\title{
Mandenkan
}

MANDENIKAN Bulletin semestriel d'études linguistiques mandé

49 | 2013

Le maninka du Niokolo

\section{La construction qualificative}

Section 13

Denis Creissels

\section{OpenEdition}

Journals

Édition électronique

URL : https://journals.openedition.org/mandenkan/609

DOI : 10.4000/mandenkan.609

ISSN : 2104-371X

Éditeur

Llacan UMR 8135 CNRS/Inalco

\section{Édition imprimée}

Date de publication : 1 juin 2013

Pagination : 93-96

ISSN : 0752-5443

Référence électronique

Denis Creissels, «La construction qualificative», Mandenkan [En ligne], 49 | 2013, mis en ligne le 25 avril 2014, consulté le 16 janvier 2023. URL : http://journals.openedition.org/mandenkan/609 ; DOI https://doi.org/10.4000/mandenkan.609

Ce document a été généré automatiquement le 16 janvier 2023.

\section{cc) (i) (2) (2)}

Creative Commons - Attribution - Pas d'Utilisation Commerciale - Partage dans les Mêmes Conditions 4.0 International - CC BY-NC-SA 4.0

https://creativecommons.org/licenses/by-nc-sa/4.0/ 


\title{
La construction qualificative
}

\section{Section 13}

\author{
Denis Creissels
}

\subsection{Remarques générales}

1 Le modifieur qualificatif succède immédiatement au nom qu'il modifie, et il ne peut en être séparé par aucun autre modifieur. En outre, selon la nature du modifieur qualificatif, il peut former avec le nom un syntagme à tonalité compacte. Enfin, on doit noter que la seule expansion possible du modifieur qualificatif est le suffixe augmentatif -baa, qui suffixé à un modifieur qualificatif constitue l'équivalent de 'très', comme dans kúntam-baa 'très sot' < kúntay 'sot' (adjectif dérivé de kún 'tête' par adjonction du suffixe privatif-ntay).

\subsection{Qualifiants non dérivés}

2 Les qualifiants non dérivés forment un syntagme à tonalité compacte totale avec le nom auquel ils succèdent. Par exemple, en (138c), si jii kando o ne constituait pas un syntagme à compacité tonale totale, on attendrait comme tonalité *jii kándǒo (cf. Jìyo kándita lée 'L'eau est chaude').

3 Les adjectifs au sens donné ici à ce terme s'utilisent typiquement comme qualifiants, mais cette fonction est aussi accessible à la plupart des verbes qualificatifs (verbes qui se construisent intransitivement avec le prédicatif ye à valeur stative, cf. 5.2), ainsi qu'à quelques verbes ordinaires et à quelques noms. A l'ex. (138), kuta est un adjectif, mé luy est un verbe ordinaire '(intr.) 'être/devenir pointu, (tr.) tailler en pointe), jaín et kaindi sont des verbes qualificatifs.

\begin{tabular}{|l|l|l|l|l|l|l|}
\hline (138) & a. & Á & ye & fee & kutaía & náati. \\
\hline & & 3SG & ACPP & calebasse & neuf.D & apporter \\
\hline
\end{tabular}


'Elle a apporté une calebasse neuve.'

\begin{tabular}{|l|l|l|l|l|l|l|}
\hline & b. & A & taða-tá & dulaa & ján-o & to. \\
\hline & & 3SG & partir-ACPP & endroit & être_loin-D & LOC \\
\hline & & \multicolumn{5}{|c|}{ 'Il est parti à un endroit éloigné.' } \\
\hline
\end{tabular}

\begin{tabular}{|l|l|l|l|l|l|l|}
\hline & c. & $\eta$ & só & jii & kand-óo & la! \\
\hline & & 1 SG & donner & eau & être_chaud-D & овL \\
\hline & & \multicolumn{4}{|c|}{ 'Donne-moi de l'eau chaude! } \\
\hline
\end{tabular}

\begin{tabular}{|c|c|c|c|}
\hline d. & Do & melu:-in-o & natiati! \\
\hline & bâton & être/devenir_pointu-D & apporter \\
\hline & \multicolumn{3}{|c|}{ Apporte un bâton pointu !' } \\
\hline
\end{tabular}

4 Comme c'est souvent le cas en mandingue, quelques verbes aptes à fonctionner comme qualifiants présentent une nasale finale uniquement dans leur emploi de qualifiant. L'ex. (139) illustre le cas de senuy 'propre', qui en tant que verbe qualificatif a la forme senu.

\begin{tabular}{|c|c|c|c|c|c|c|}
\hline (139) & a. & $\tilde{N} i^{i}=\underline{n}$ & feive & ye & senu & lerie. \\
\hline & & DEM & calebasse-D & ACPP & être_propre & FOC \\
\hline & & \multicolumn{5}{|c|}{ 'Cette calebasse est propre.' } \\
\hline
\end{tabular}

\begin{tabular}{|l|l|l|l|l|l|l|l|}
\hline & b. & Luu & senún-o & lú & felé:ée & ye & dii. \\
\hline & & maison & être_propre-D & PL & regarder.D & ACPP & être_agréable \\
\hline & & \multicolumn{5}{|l|}{ 'C'est agréable de voir des maisons propres.' } \\
\hline
\end{tabular}

\subsection{Le suffixe sélectif -maa}

5 Le suffixe -maa peut de manière générale s'ajouter aux qualifiants non dérivés avec une valeur sélective ('dans une situation donnée, le $\mathrm{N}$ qui est le seul à posséder la qualité en question, ou celui qui la possède au plus haut degré') : fee kuta-máa 'la calebasse qui est neuve (parmi un ensemble de calebasses dont certaines sont usagées)', jii kandi-máa 
'l'eau la plus chaude (dans une situation où sont présents plusieurs récipients contenant de l'eau à des températures différentes)'.

6 Toutefois avec les verbes qualificatifs, -maa a aussi un emploi purement syntaxique, car sa présence est nécessaire pour qu'un verbe qualificatif puisse s'utiliser nominalement pour exprimer une qualification se rapportant à un nom sous-entendu. Par exemple, $k a$ indo ne peut pas s'utiliser nominalement pour signifier 'un(e) qui est chaud(e)'. Seule la forme kaindima peut s'utiliser nominalement avec cette valeur, et dans ce cas, -maa n'implique pas une valeur sélective.

7 En outre, quelques verbes qualificatifs (dii 'être agréable, facile', fee 'être léger', kuu 'être mauvais', ñin 'être beau, bien' et see 'être fade') ne peuvent s'utiliser comme qualifiants que pourvus du suffixe sélectif -maa, même en présence du nom qualifié. Avec ces lexèmes, de manière générale, ce suffixe n'a donc pas les implications sémantiques qu'il a avec les autres lexèmes auxquels il s'attache.

\subsection{Qualifiants dérivés}

8 Les suffixes permettant de dériver des adjectifs ont été énumérés en 6.3.2. Les qualifiants ainsi formés occupent la même position que les qualifiants non dérivés, mais n'ont pas les mêmes propriétés tonales. La règle générale est que les qualifiants dérivés forment avec le nom qu'ils modifient un syntagme à compacité tonale partielle: ils maintiennent leur ton lexical, mais il n'apparait jamais de ton haut démarcatif à la finale $\mathrm{du}$ nom, et si le nom a un schème tonal non-canonique (comme par exemple kupkuwu ilu 'chacal' ou bara insay 'acacia albida'), il perd son ton haut non-initial :

\begin{tabular}{|c|c|c|}
\hline dindiy málibali & 'enfant effronté' & < málu 'avoir honte' \\
\hline súbu sántaa & 'viande mise en vente' & < sáy 'acheter \\
\hline dindiy hakilimaa & 'enfant intelligent' & < hakili 'intelligence' \\
\hline dindin balándin & 'enfant désobéissant' & < balay 'désobéir' \\
\hline kuykuwulu foñontay & 'chacal sans queue' & < foño 'queue' \\
\hline baransay kéturiy & 'acacia albida abattu' & < kétu 'abattre' \\
\hline
\end{tabular}

Les ordinaux en -ñjay font toutefois exception à cette règle, et se comportent tonalement comme les qualifiants non dérivés. L'ex. (140) montre que fú-ílañjay 'deuxième' perd dans la construction qualificative le ton haut lexical qu'il manifeste en dehors de cette construction.

\begin{tabular}{|c|c|c|c|c|}
\hline (140) & a. & Sugkutu & fula-ñja:-n-o & náa-ta. \\
\hline & & fille & deux-ORD-D & venir-ACPP \\
\hline & & \multicolumn{3}{|c|}{ ‘La deuxième fille est revenue.’ } \\
\hline
\end{tabular}




\begin{tabular}{|l|l|l|l|}
\hline & b. & Fui-íla-ñjaíín-o & náa-ta. \\
\hline & & deux-ORD-D & venir-ACPP \\
\hline & & \multicolumn{2}{|c|}{ 'La deuxième est revenue.' } \\
\hline
\end{tabular}

\subsection{Qualifiants composés}

En se postposant simplement à un nom, sans matériau morphologique additionnel, les syntagmes qualificatifs ainsi que les syntagmes à modifieur numéral fonctionnent comme qualifiants de sens ornatif ('pourvu de'), c'est-à-dire avec le sens encodé en règle générale par le dérivatif -maa. Comme les exemples suivants l'illustrent, ces qualifiants composés forment avec le nom qu'ils modifient un syntagme à compacité tonale partielle :

\begin{tabular}{|c|c|}
\hline Dindiy bulu diimaa & 'enfant habile' (bulu 'main', diimaa ‘bon') \\
\hline dindiy sínkoto jay & 'enfant aux longues jambes' (sínkoto 'dessous de la jambe', jáy 'être long') \\
\hline gidi daa fúla & 'fusil à deux coups' (daa 'ouverture', fúla 'deux’) \\
\hline sáayajizi síy sába & ‘bélier à trois pattes’ (síi ‘patte, sába ‘trois’) \\
\hline
\end{tabular}

\section{AUTEUR}

DENIS CREISSELS

Université de Lyon

Denis.Creissels@univ-lyon2.fr 